\title{
Innovative use of Intralipid in Critical Care Setting
}

\author{
Muzzammil Ali ${ }^{1}$ and Vikram Anumakonda ${ }^{2 *}$ \\ ${ }^{1}$ Department of Intensive Care Medicine, Dudley Group Hospital NHS Foundation Trust, UK \\ ${ }^{2}$ University of Birmingham, $U K$
}

Submission: August 01, 2017; Published: August 16, 2017

*Corresponding author: Vikram Anumakonda, Consultant Physician, Critical and Acute care, Dudley Group Hospital NHS Foundation, Pensnett Road, Dudley, West Midlands, UK DY1 2HQ Senior Honorary Clinical Lecturer, University of Birmingham, UK;

Email: vikramanumakonda@gmail.com

\section{Case Series}

\section{Case 1: Refractory hypotension to vasopressors in a case of mixed overdose}

A 42-year-old gentleman presented to hospital emergency department (ED), following a deliberate self-harm with an intentional overdose of $75 \mathrm{mg}$ Ramipril, 450mg Amlodipine and $10 \mathrm{mg}$ Penicillamine. His initial toxicology screen was negative for paracetamol, salicylate, alcohol or illicit drug. He had a single functioning left kidney, on background of hypertension, cysteinuria, chronic kidney disease (baseline creatinine 130150).

On presentation to the resuscitation unit, he was normocardic $(60 /$ minute), hypotensive $(88 / 41 \mathrm{mmHg})$. His GCS was 15 . He was oliguric. His preliminary blood tests revealed new onset acute kidney injury. His ECG showed normal sinus rhythm with a normal QTc. Blood gas analysis revealed compensated metabolic acidosis; $\mathrm{pH}$ and lactate were within normal limits, but his base excess was -3.7 .

Despite intensive fluid resuscitation and administration of glucagon and calcium chloride to antagonise calcium channel blocker in the resuscitation department, he remained persistently hypotensive and oliguric. He only passed $30 \mathrm{mls}$ of

urine during his 8 hours of being in hospital, and his base excess was static around -3.0.

He was admitted to the intensive care unit for vasopressor support, with an intent of improve blood perfusion to his only kidney, to mitigate further insult to his remaining renal function. He was started on noradrenaline with a target invasive mean arterial blood pressure (MAP) of $65-70 \mathrm{mmHg}$. However, his MAP did not respond to maximum dose of noradrenaline and crystalloids. In view of his deliberate over dose, the National
Inpatient Poisons Service (NPIS) advice was sought. They advised to start patient on insulin infusion with back ground $10 \%$ glucose infusion and supportive therapy.

At 12.00 hours, day 2 of admission to ICU, patient started to develop severe hypoglycaemia despite being on continuous infusion of $50 \%$ glucose infusion via a central venous catheter. He started to become more hemodynamically unstable. At 2.00 A.M., despite adrenaline boluses at 5, 10 and 15 micrograms, his MAP was $50 \mathrm{~mm} \mathrm{Hg}$. Moreover, these adrenaline boluses propensiated further severe hypotension with systolic blood pressures from invasive monitoring recording only $55 \mathrm{~mm} \mathrm{Hg}$. His base excess had also become more negative from -3.7 to -5.5 in spite of constant infusion of $8.4 \%$ sodium bicarbonate.

A further advice was sought from the NPIS, who advised to continue with a high dose insulin infusion and symptomatic support. Despite being on a high dose insulin infusion with appropriate potassium and glucose supplementation, patient cardiovascular deterioration continued. His glucose levels were below 4 mill moles/liter, despite constant infusion of $50 \%$ dextrose infusion due to insulin infusion. Interestingly, his neurology remained intact, GCS15 (E4M5V6) all throughout. Intralipid rescue therapy was initiated with the consent of patient. A trial of Intralipid $20 \% 400 \mathrm{mls}$ over 1 hour was commenced following an initial bolus of $100 \mathrm{ml}$ (Figures 1 \& 2) at 3.00 A.M. (Figure 1).

During and following Intralipid infusion, his hemo-dynamic status dramatically improved. His blood pressure had improved to $112 / 55 \mathrm{~mm} \mathrm{Hg}$ and MAP $74 \mathrm{~mm} \mathrm{Hg}$. His base excess had improved from -5.1 to -1.0 (Figure 2). His urine output had also improved dramatically to $150 \mathrm{ml} / \mathrm{hr}$. His insulin infusion was successfully weaned off within an hour of Intralipid therapy. He 
remained hemodynamically stable for next 24 hours. He was subsequently discharges from the intensive care department, and his repeat bloods showed an improvement in his renal function (back to his baseline at 127).

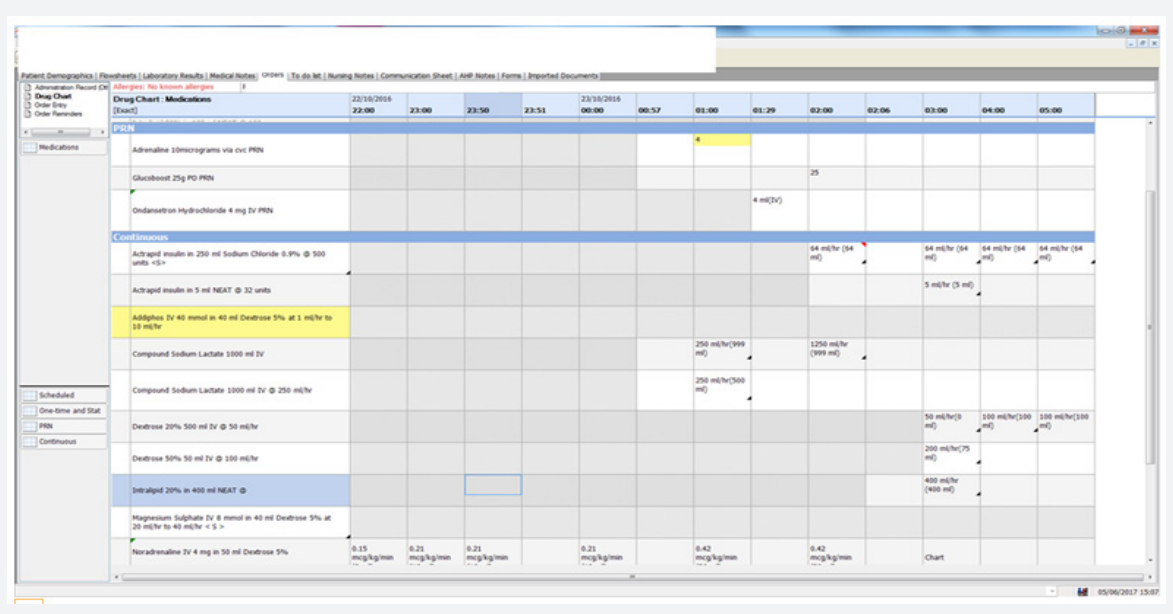

Figure 1: Case 1 demonstrates Intralipid infusion over an hour for the patient with mixed overdose (Amlodipine, Ramipril and Pencillamine).

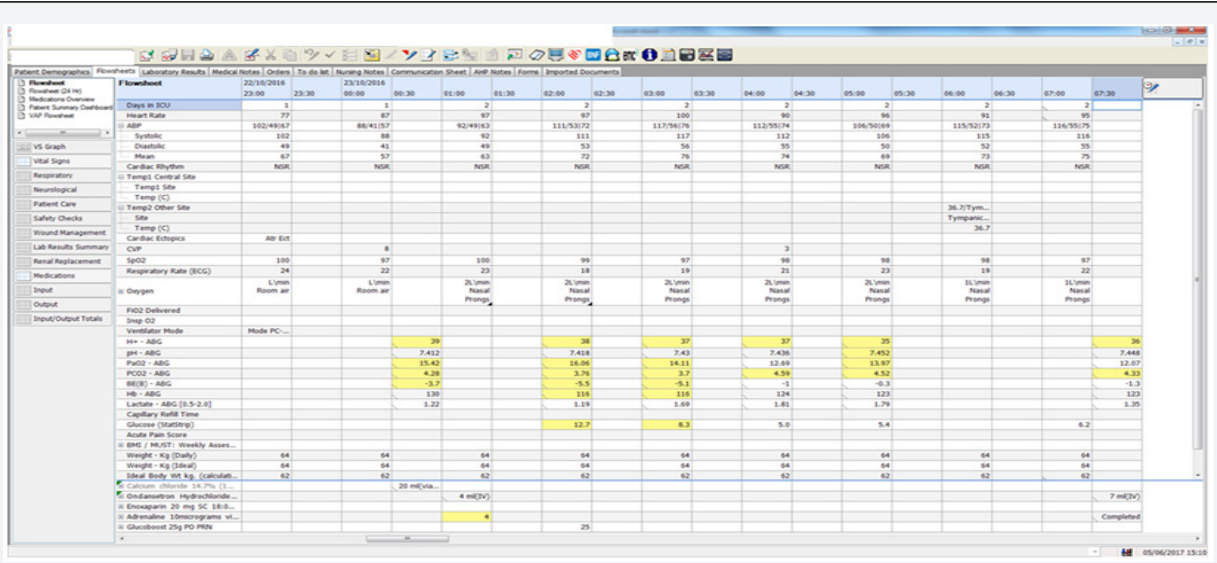

Figure 2: Case 1 demonstrates hemodynamic instability responding to Intralipid for the patient with mixed overdose (Amlodipine, Ramipril \& Pencillamine) with hourly observations.

\section{Case 2: Haemodynamic instability following a mixed overdose}

A 60-year-old female with multiple sclerosis presented with a GCS of 3 following an overdose of gabapentin and tramadol. She had a background of asthma, multiple sclerosis (wheelchair bound) and depression. A supra-glottic device (igel) was inserted by the paramedics on site to secure her airway. Once in the resuscitation department, an endotracheal intubation using a size 7 with subglottic suction port was used to secure a definitive airway. All medical imaging including computer tomography (CT) of her head and neck were unremarkable. Paracetamol, salicylate, carbamazepine and ethanol levels were undetectable as a part of initial toxicology screen. Gastric lavage with activated charcoal was tried, but with little effect.

She was transferred to the intensive care unit for supportive treatment. She was bradycardic and hypotensive. Her 12 lead electrocardiograph (ECG) showed sinus bradycardia with a normal QTc. Blood gas analysis revealed metabolic acidosis with an elevated lactate; pH 7.323, base excess -5.4, lactate 2.40. Her routine biochemistry and full blood count and coagulation screen were unremarkable.

Despite aggressive fluid resuscitation, noradrenaline and an isoprenaline infusion, she remained severely hypotension (85/59mm $\mathrm{Hg}$ ) and bradycardic with QT interval prolongation (HR $<45 /$ minute). Although her serial blood gases improved following these interventions, she persistently remained haemodynamically unstable with increased propensity to arrhythmias. She required regular intermittent boluses of adrenaline for blood pressure and heart rate support. Her GCS remained at 3 despite stopping her sedation (Figure 3-5).

In view of her hemodynamic instability leading to periarrest and low GCS, despite optimal therapy, Intralipid rescue 
therapy was initiated. Intralipid 20\% $100 \mathrm{mls}$ bolus over 1 minute followed by as an infusion was started. Following this, her blood pressure had increased linearly and so did her heart rate. As her heart rate and blood pressure improved dramatically to $70-85 \mathrm{bpm}$ and $203 / 103 \mathrm{~mm} \mathrm{Hg}$ respectively, her inotropes support started to decline within an hour of infusion of Intralipid (Figures 3-5).

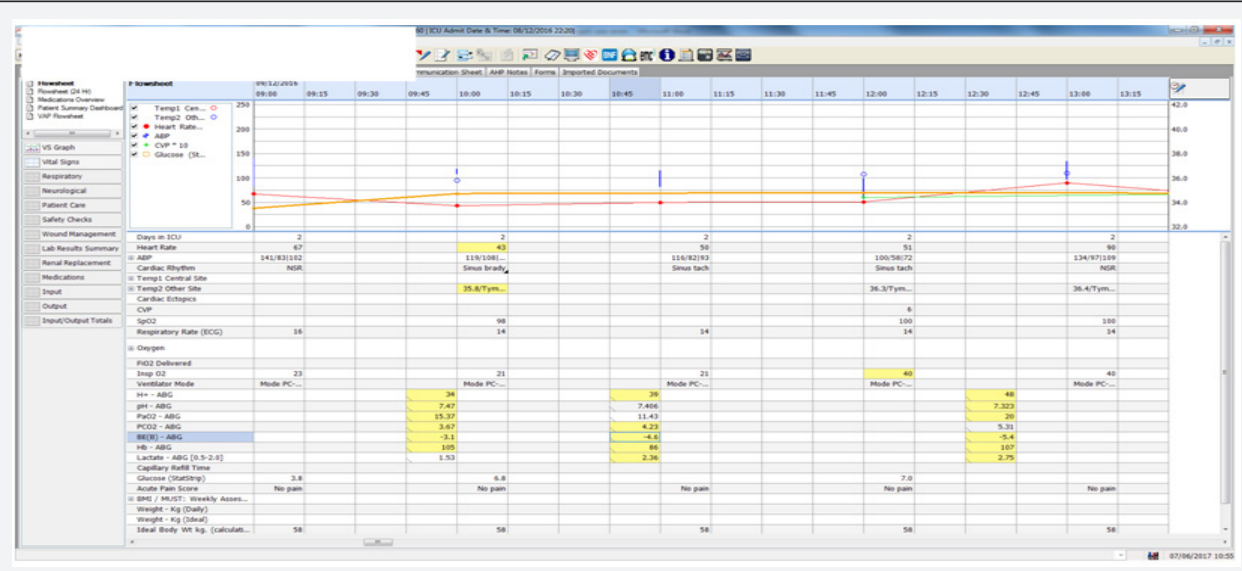

Figure 3: Demonstrates hemodynamic instability responding to Intralipid for the MS patient with mixed overdose (Gabapentin \& tramadol) MS patient in haemodynamic instability in her case despite using adrenaline: Intra-lipid rescue therapy.

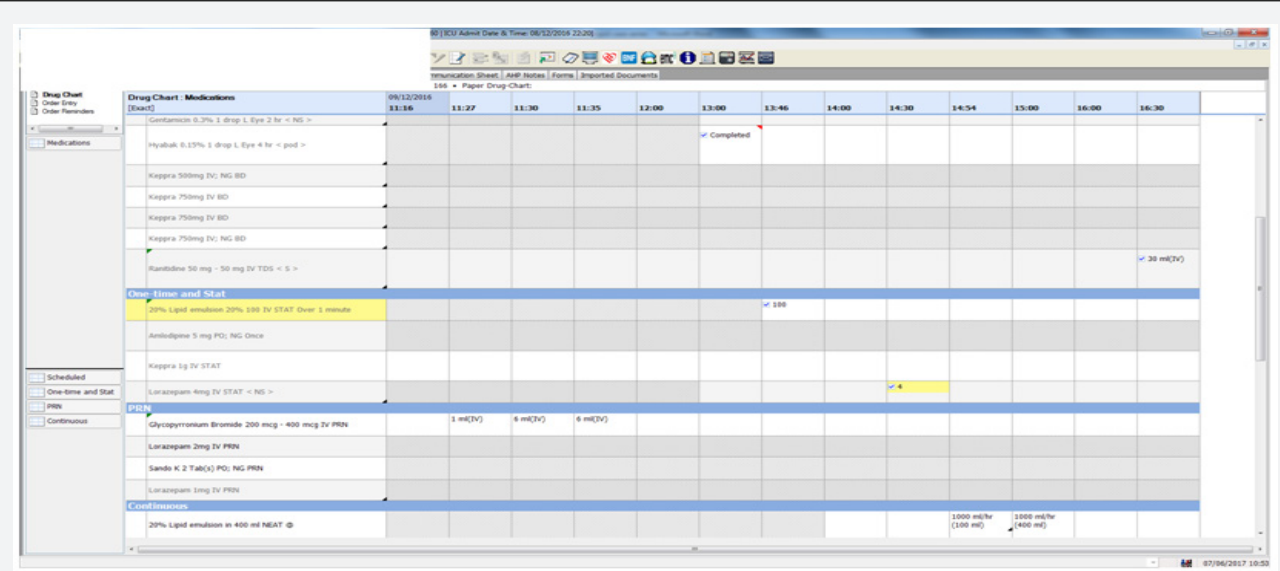

Figure 4: Demonstrates timing and mode of administration of Intra-lipid rescue bolus to the MS patient with mixed overdose (Gabapentin \& tramadol) MS patient in haemodynamic instability in her case despite using adrenaline: Intra-lipid rescue therapy.

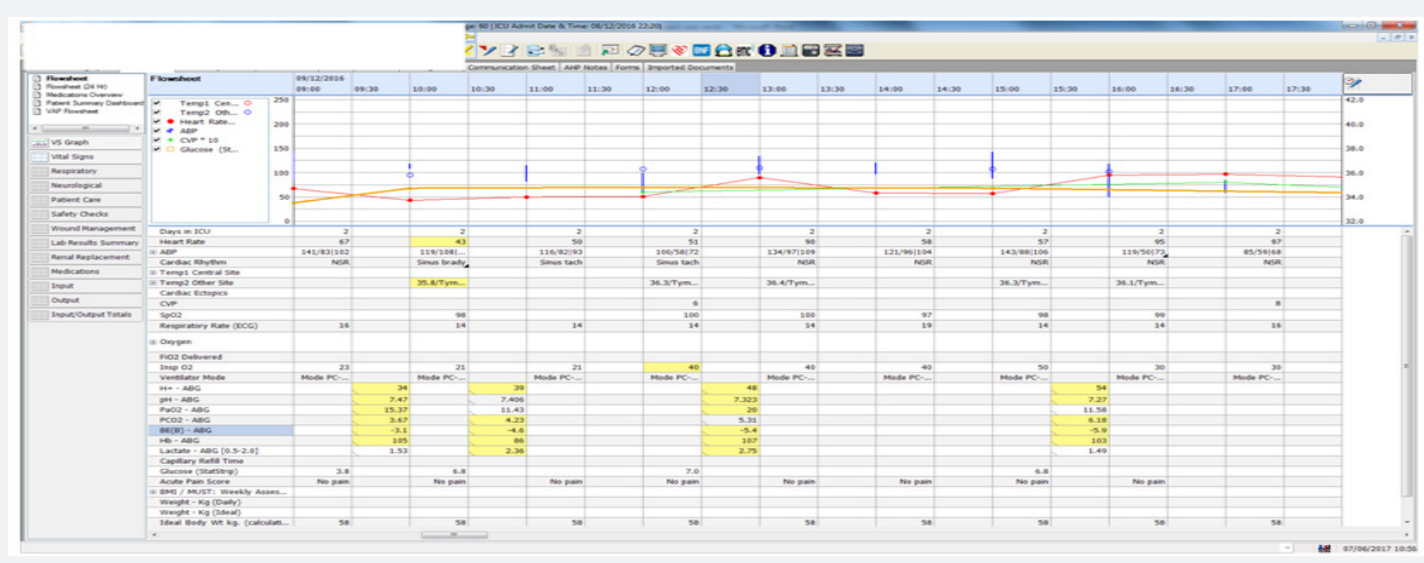

Figure 5: Demonstrates hemodynamic stability after Intra-lipid rescue therapy of the MS patient with mixed overdose (Gabapentin \& tramadol) with hourly observations. 
Following morning, patient did not regain neurological response during traditional sedation break, as part of morning consultant ward round. As it was not possible to clinically possible to rule out status epilepticus, she was empirically started. Following neurologist advice, levetiracetam was deemed a safest antiepileptic in her case. Her anti-epileptics were discontinued after 48-72 hours, as patient started appropriate neurological response. Later on, her inotropes were successfully weaned off.

On day 5, she was extubate and spontaneous ventilation. Her blood pressure and heart rate were self-maintained within normal limits (BP 125/61 mmHg). She was neurologically intact on day 5, with no further seizures. Following extubating, she was able to confirm her intentional deliberate overdose medication and quantity taken.

\section{Discussion}

Intralipid is an emulsion of soybean oil, egg phospholipids and glycerine. This emulsion had a lot of attention recently owing to its clinical application to treat local anaesthetic systemic toxicity (LAST) and non-local anaesthetic drug toxicity.

Numerous laboratory studies have shown that Intralipid can prevent and/or improve the efficacy of resuscitation from cardiovascular collapse caused by iatrogenic severe bupivacaine overdose in rats and dogs. Subsequent case reports have alluded to this, but less robustly than these laboratory studies.

Rosenblatt et al. for example achieved ROSC in a patient who developed a cardiac arrest induced by mepivacaine and bupivacaine (peripheral nerve block) when standard resuscitation efforts failed by administering a $100 \mathrm{ml}$ bolus of Intralipid. This patient recovered completely with no neurological deficit or cardiovascular sequelae [1]. Furthermore, McCutchen and Gerancher found that the use of Intralipid attenuated and prevented the progression of local anaesthetic cardiac and neurological toxicity in a patient who developed seizures and VT following a combined femoral catheter (ropivacaine) and sciatic (bupivacaine) block [2]. This was supported by Foxall et al who found that Intralipid can reverse neurological signs and symptoms of LAST, including seizures and altered mental state [3]. There is therefore some suggestion that Intralipid administration can improve cardiovascular and neurological outcomes in LAST.

Case reports have also shown a benefit of Intralipid in treating overdose of non-local anaesthetic drug toxicity including in mixed overdose; when competitive inhibition of carrier proteins including albumin or lipophilic drugs were involved. Sirianni et al. for example saw the success of using Intralipid in a patient who incurred a cardiac arrest following an intentional overdose of bupropion and lamotrigine. Despite 90min of VT/VF/PEA, conventional interventions were futile. However, when a single bolus of lipid emulsion was administered, the patient recovered normal vital signs and ultimately left the hospital with no major neurological deficit [4]. Some studies have also found that in the treatment of mixed drug overdose with lipid emulsion, there was a reduction in the incidence of intubation and a shorter duration of stay in the intensive care unit than matched controls not receiving lipid [5].

There are three possible theoretical areas where Intralipid can exert its effects-intravascularly, intercellularly and upon membrane channels.

1. Intravascularly, Intralipid functions as a lipid sink. The intravascular lipid mass binds to a particular drug (drug administered in overdose) to a sufficient degree that it pulls it away from its target tissue, thereby reversing the toxicity [5].

2. Intercellularly, Intralipid can modify metabolic and signaling pathways, and can also exhibit a direct cardiotonic effect. The latter has been seen in rodent hearts. Anesthetic agents like bupivacaine can inhibit fatty acid metabolism, which can compromise the heart's preferred energy substrate, resulting in bradycardia and cardiac arrest. An Intralipid bolus can offset this potent inhibition, with the subsequent additional benefit of suppressing mitochondrial permeability there by preventing myocardial apoptosis [6].

3. When Intralipid acts upon membrane channels, it can modulate cardiac sodium channels to reduce the bupivacaine inhibition of sodium channel currents. This can reduce bupivacaine toxicity and improve cardiac outcomes [7]. It can also attenuate ischemia reperfusion injury by activating the Akt cascade leading to the inhibition of GSK3 -beta, which has been implicated in preventing myocardial ischemia-reperfusion injury [8].

Unlike the aforementioned case reports, these two cases highlight potential innovative successful use of Intralipid as a rescue therapy in human beings even at delayed stages of poisoning. These two cases largely support the intravascular and intercellular theories of Intralipid action and are the first of their kind in existing literature.

Case 1 describes the clinical dilemma whereby high blood concentrations of the lipid-soluble antihypertensive, Ramipril and amlodipine, made treating the resulting hypotension very difficult. Vasopressor infusions and boluses in high concentrations achieved no benefit in improving blood pressure or urine output. However, when an Intralipid infusion was commenced, blood pressure, urine output and base excess all improved dramatically.

This resulting outcome supports the lipid sink hypothesis of Intralipid action [5]. The intravascular presence of lipid emulsion acted as a reservoir to adsorb both Ramipril and amlodipine to a sufficient extent that they were not able to inhibit ACE or calcium 
channels respectively. There by allowed the vasopressors to act of their respective alpha and beta receptors. Subsequently vasopressors were being weaned, as physiological innate control was established leading to improve blood pressure. There could be a likely competitive displacement of albumin amlodipine complex by lipids during Intralipid rescue therapy.

Case 2 describes the clinical scenario whereby haemodynamic instability and altered mental status arose when high blood concentrations of the lipid soluble drugs, gabapentin and tramadol, were present. Thus, high blood concentrations lead to haemodynamic instability in spite of vasopressors and inotropic support.

Intralipid infusion served as a lipid sink, [5] allowing noradrenaline and isoprenaline to act on their respective alpha and beta receptors to improve cardiovascular stability. Furthermore Intralipid may have had a direct cardiotonic effect in improving heart rate [6]. Furthermore it may have promoted a cytoprotective benefit in preserving fatty acid oxidation for cardiac myocytes and inhibiting cardiac apopotosis [6]. Although it is unclear whether the positive neurological outcome seen was attributable to keppra or Intralipid as EEG studies were not done, it is befitting to propose that Intralipid may have had an role in reversing this patient's adverse neurological outcomes. This is consistent with that described by [3] and many other case reports with reversed adverse neurological outcomes.

Whilst all that has been described points in a positive direction, it is worthwhile mentioning that Intralipid has its downsides. Reports exist of pulmonary complications in neonates when used in high concentrations for parenteral nutrition [9], hyperamylasaemia without symptoms of pancreatitis following successful lipid rescue from cardiac arrest due to bupivacaine [10], and cardiovascular instability post-infusion of Intralipid which required meticulous monitoring [10]. However, in both our cases described, these downsides were not experienced.
The proven indications for Intralipid in humans beyond the scope of LAST is yet to be confirmed and its mechanism of action in these contexts still requires to be researched. However, our cases demonstrate Intralipid's future potential as a rescue therapy and its promising role in saving many lives.

\section{Reference}

1. Rosenblatt MA, Abel M, Fischer GW, Itzkovich CJ, Eisenkraft JB (2006) Successful use of a $20 \%$ lipid emulsion to resuscitate a patient after a presumed bupivacaine-related cardiac arrest. Anesthesiology 105(1): 217-218.

2. McCutchen T, Gerancher JC (2008) Early Intralipid therapy may have prevented bupivacaine-associated cardiac arrest. Reg Anesth Pain Med 33(2): 178-180.

3. Foxall G, McCahon R, Lamb J, Hardman JG, Bedforth NM (2007) Levobupivacaine-induced seizures and cardiovascular collapse treated with Intralipid. Anaesthesia 62(5): 516-518.

4. Sirianni AJ, Osterhoudt KC, Calello DP, Muller AA, Waterhouse MR (2008) Use of lipid emulsion in the resuscitation of a patient with prolonged cardiovascular collapse after overdose of bupropion and lamotrigine. Ann Emerg Med 51(4): 412-415.

5. Weinberg G (2012) Lipid Emulsion Infusion: Resuscitation for Local Anesthetic and other Drug Overdose. Anesthesiology 117(1): 180-187.

6. West PL, McKeown NJ, Hendrickson RG (2010) Iatrogenic lipid emulsion overdose in a case of amlodipine poisoning. Clin Toxicol (Phila) 48(4): 393-396.

7. Marwick PC, Levin AI, Coetzee AR (2009) Recurrence of cardiotoxicity after lipid rescue from bupivacaine-induced cardiac arrest. Anesth Anal 108(4): 1344-1346.

8. Partownavid P, Umar S, Li J, Rahman S, Eghbali M (2012) Fatty acid oxidation and calcium homeostasis are involved in the rescue of bupivacaine induced cardiotoxicity by lipid emulsion in rats. Crit Care Med 40(8): 2431-3437.

9. Mottram AR, Valdivia CR, Makielski JC (2011) Fatty acids antagonize bupivacaine-induced I(Na) blockade. Clinical toxicology 49(8): 729733.

10. Rahman S, Li J, Bopassa JC, Umar S, Iorga A, et al. (2011) Phosphorylation of GSK-3beta mediates Intralipid-induced cardioprotection against ischemia/reperfusion injury. Anesthesiology 115(2): 242-253.

\section{Your next submission with Juniper Publishers} will reach you the below assets

- Quality Editorial service

- Swift Peer Review

- Reprints availability

- E-prints Service

- Manuscript Podcast for convenient understanding

- Global attainment for your research

- Manuscript accessibility in different formats ( Pdf, E-pub, Full Text, Audio)

- Unceasing customer service

Track the below URL for one-step submission https://juniperpublishers.com/online-submission.php 